\title{
An Experimental Study of the Dependence on the Current Intensity of the Erosion of Thoriated Tungsten Cathodes in Plasma Arcs
}

\author{
Eduardo Casado, Vicente Colomer, Rafael Sicilia, and Encarnación Muñoz-Serrano
}

\begin{abstract}
The erosion occurring in thoriated tungsten cathodes used in transferred plasma torches operating with current intensities ranging from 30 to $210 \mathrm{~A}$ has been experimentally investigated. The cathode erosion rate was determined by measuring the cathode's weight loss after arcing, and the eroded cathode surface was explored by scanning electron microscope and energy dispersive $X$-ray spectrometer. It has been discovered that a critical current intensity, $I_{C R} \sim 50 \mathrm{~A}$, exists for which the cathode erosion rate becomes maximum.
\end{abstract}

Index Terms-Arc plasma, cathode erosion, thoriated tungsten, $\mathrm{W}-\mathrm{ThO}_{2}$ cathode.

\section{INTRODUCTION}

C ATHODE erosion is still a problem hindering the widespread application of thermal plasmas in industry [1]-[3]. This problem is of particular importance in thermionic-emission cathodes due to the high temperatures attained on their spots. In order to reduce the working temperature, it has become common to use binary thermionic cathodes, which combines the high fusion point of the base refractory material with the low work function of the dopant. The most commonly employed cathodes of this type are thoriated tungsten cathodes. However, the erosion problem in these cathodes continues to be a basic question which has been intensively investigated in recent years.

Presently, cathode erosion has been theoretically studied by various researchers using models based on the balance of energy flux and charged particles between the spot and the near-cathode region [4]-[8]. In the case of thoriated tungsten cathodes, it is necessary to take into account in the models that the cathode work function depends on the degree of spot coverage by the thorium atoms [9], which is determined by the processes of desorption and adsorption of thorium on the spot and by the diffusion of thorium from the inside of the cathode to its surface [10].

The influence of these processes on the lifetime of the cathode was studied by Nemchinsky [11] assuming a linear distribution of temperature on the cathode, and more generally by Casado and Colomer [12], who developed a numerical model that did

\footnotetext{
Manuscript received March 5, 2001; revised July 27, 2001. This work was supported in part by IBERDROLA S. A. under the PLASMATEK Project.

E. Casado and V. Colomer are with the Department of Physics, Cordoba University, Cordoba 14071, Spain (e-mail: fa1caree@uco.es).

R. Sicilia and E. Muñoz-Serrano are with the Laboratory of Plasma Technology, Cordoba University, Cordoba 14071, Spain.

Publisher Item Identifier S 0093-3813(01)11210-5.
}

not fix a priori the temperature distribution on the cathode and took into account the Joule heating of the cathode.

In the latter study, it was found that a critical value of the current intensity exists for which the lifetime of the thoriated tungsten cathode has a minimum value. Since that lifetime is essentially tied to cathode erosion, this result suggests that in thoriated tungsten cathodes, the cathode erosion will be maximum for a certain critical value of the current intensity. The experimental corroboration of this prediction motivated the present paper. To the best of our knowledge, although thoriated tungsten cathodes have been the subject of intense experimental investigation in recent years [13]-[18], no experimental data supporting or rejecting the mentioned prediction has been reported.

In [13], an experimental study of the influence of the cathode geometry and of the plasma-forming gas composition on erosion of the thoriated tungsten cathodes was carried out, but at a constant current intensity of $200 \mathrm{~A}$.

The factors affecting erosion in thoriated tungsten cathodes have been experimentally studied by Zhou and Heberlein [14], [15] by means of spectrometry, pyrometry, high-speed photography, scanning electron microscope (SEM), and energy dispersive X-ray spectrometer (EDX). They determined the electron temperature and electron number density in the cathode region for current intensities ranging from 80 to $200 \mathrm{~A}$. They also measured the temperature distribution on the cathode for current intensities between 100 and $300 \mathrm{~A}$. Nevertheless, they did not report data on the dependency of erosion on current intensity. The measurements of cathode mass loss reported by them were made with a single current intensity of $200 \mathrm{~A}$.

The performances of thoriated tungsten cathodes and tungsten cathodes doped with other rare-earth elements were compared in [16] on the basis of their surface temperatures, but a constant arc current of 200 A was used for arcing. Although surface temperature profiles for thoriated tungsten cathodes with various arc currents were presented in [17], no relation with the cathode erosion rate was established. In [18], a novel tungsten cathode activated with nanoscale thoria was investigated, but no data on the erosion rate was reported.

In the present paper, the dependence of the erosion rate of thoriated tungsten cathodes on the current intensity of the arc is studied with currents ranging from 30 to $210 \mathrm{~A}$. In the next section, the experimental procedures and the required precautions to guarantee the reliability of our erosion measurements are described. We report and discuss our results in SectionIII and SectionIV, respectively. In the end, our conclusions are drawn. 


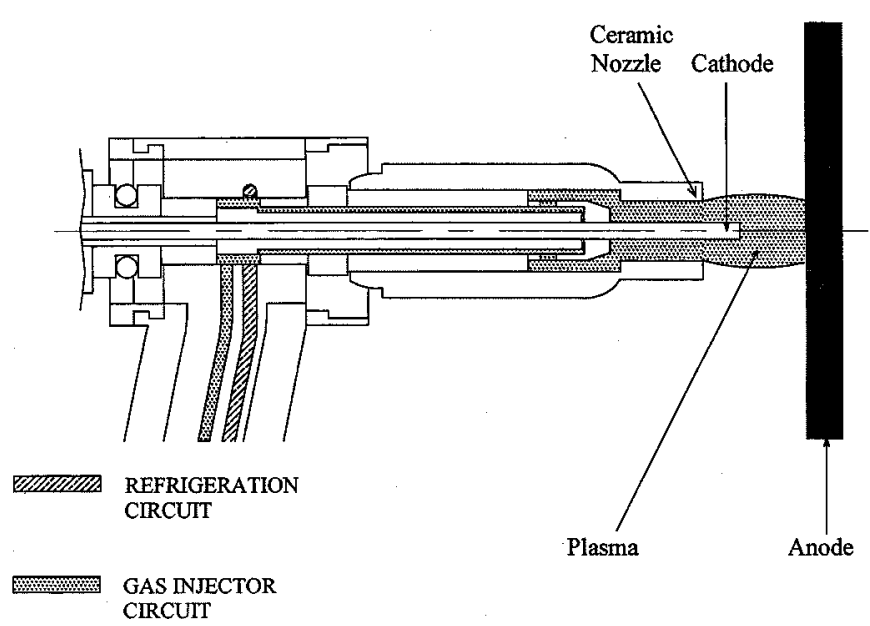

Fig. 1. Schematic diagram of the plasma torch used.

\section{EXPERIMENTAL PROCEDURES}

Cylindrical cathodes of thoriated tungsten $(\mathrm{W}+2 \mathrm{wt} \%$ $\mathrm{ThO}_{2}$ ) with a flat tip and a diameter of $2.4 \mathrm{~mm}$ were used on a de transferred torch (model TIG-350 DC-P) at atmospheric pressure. Cathodes with a flat tip are commonly used with this type of torch in industrial applications. To reproduce as similarly as possible the industrial condition of the torch's use, we have kept the cathodes with a flat tip, which is the shape they have commercially. This does not affect the stability of the arc, which was totally stable in the range of current that we used.

The working conditions of the arc were the normal ones used in industrial applications, with plasma gas being injected between the protective ceramic nozzle and the cathode, and then activating the arc between the cathode and a graphite anode situated in front (see Fig. 1). All of this is done in the open air. We chose a graphite anode because of its refractory properties. The graphite anode does not need cooling, which permits us to work with a very simple experimental setup.

Different current intensities were used for the arc, sweeping the current interval from 30 to $210 \mathrm{~A}$. The cathode-anode distance was always finely adjusted so that the voltage of the arc was $22 \mathrm{~V}$ in all cases. For each one of the current intensities used, the cathode was weighed before and after arcing on an electronic balance (COBOS TR104) with a precision of $0.1 \mathrm{mg}$. In this way, the cathode mass loss was determined, which, along with the value of the current intensity and the arcing time, permitted the calculation of the cathode erosion rate, defined as the ratio between the loss in mass and the total charge that passed through the cathode.

The cathode surface before and after arcing was explored by SEM and EDX, with which a study of the erosion effects that complement the mass loss measurement was done.

Initially the plasma-forming gas used was pure argon. Once the arc was interrupted, the cathode remained at a very high temperature immersed in the air. After arcing, a dark ring-shaped layer was observed in the cathode far away from the cathode tip. The EDX analysis of this layer showed it to have a high oxygen content which indicated the formation of oxide on the cathode surface (see Fig. 2), probably the brown oxide $\mathrm{WO}_{2}$

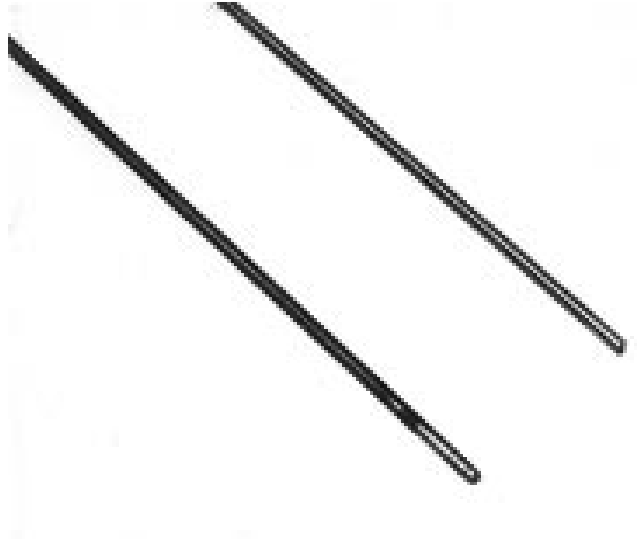

Fig. 2. Cathodes after arcing with a mixture of argon and hydrogen (cathode at top of the photograph) or with pure argon (cathode at bottom of the photograph) as the plasma-forming gas. A ring-shaped layer of oxide at $12 \mathrm{~mm}$ from cathode's tip can be seen in the latter cathode. The EDX analysis indicated the following atomic composition at a point on this layer: $34.34 \% \mathrm{O}$, $0.24 \% \mathrm{Th}, 20.18 \% \mathrm{~W}$, and $45.24 \% \mathrm{C}$. Off the layer the composition is: $25.45 \%$ $\mathrm{O}, 0.32 \% \mathrm{Th}, 24.15 \% \mathrm{~W}$, and $50.08 \% \mathrm{C}$ at a point closer to the tip, and $22.88 \%$ $\mathrm{O}, 0.51 \% \mathrm{Th}, 17.64 \% \mathrm{~W}$, and $58.97 \% \mathrm{C}$ at a point further away from the tip. The EDX analysis of the lateral surface of the cathode used with a mixture of argon and hydrogen gave the following atomic composition: $2.89 \% \mathrm{O}, 1.74 \%$ $\mathrm{Th}, 86.88 \% \mathrm{~W}$, and $8.49 \% \mathrm{C}$.

with a boiling point of $2273 \mathrm{~K}$ [19]. The formation of a tungsten oxide layer was also observed in [17], where it was found that a small contamination of the plasma-forming gas with oxygen strongly influenced the cathode performance. The formation of the oxide layer in our experiments was highly undesirable as it could distort the erosion measurements that we made on the basis of variation of cathode mass.

Since the layer of oxide formed in this way had to mainly originate upon the deactivation of the arc, when the cathode is left still incandescent in the presence of the atmospheric oxygen, the plasma-forming gas flow was maintained for 5 min once the arc was turned off. In this way, the cathode was protected by an argon shroud until it was cool enough.

In spite of this precaution, as the arcing took place in the open air the atmospheric oxygen could still contaminate the argon and reach the cathode surface during arcing. To avoid this, we introduced hydrogen into the plasma-forming gas, hoping that the strong reducing character of hydrogen would prevent the contaminating oxygen from reacting with the cathode surface to produce the undesirable oxide layer.

For this reason, a mixture of argon (99.9995\% pure) with a $2 \%$ volume of hydrogen and a flux of $20 \mathrm{~L} / \mathrm{min}$ was used as plasma-forming gas. With this plasma-forming gas and maintaining the gas flow for 5 min after shutting off the arc, no formation of the oxide ring was observed (see Fig. 2) and the measurements of the cathode's weight loss were reproducible without any significant dispersion. This guarantees the reliability of the erosion measurements that are shown in the following section, which are also supported by the SEM examinations of the cathode surface.

On the other hand, the mixture of argon with a $2 \%$ of hydrogen is an industrially important plasma-forming gas, due to the fact that the addition of hydrogen to argon permits reaching higher temperatures in the arc [20]. 


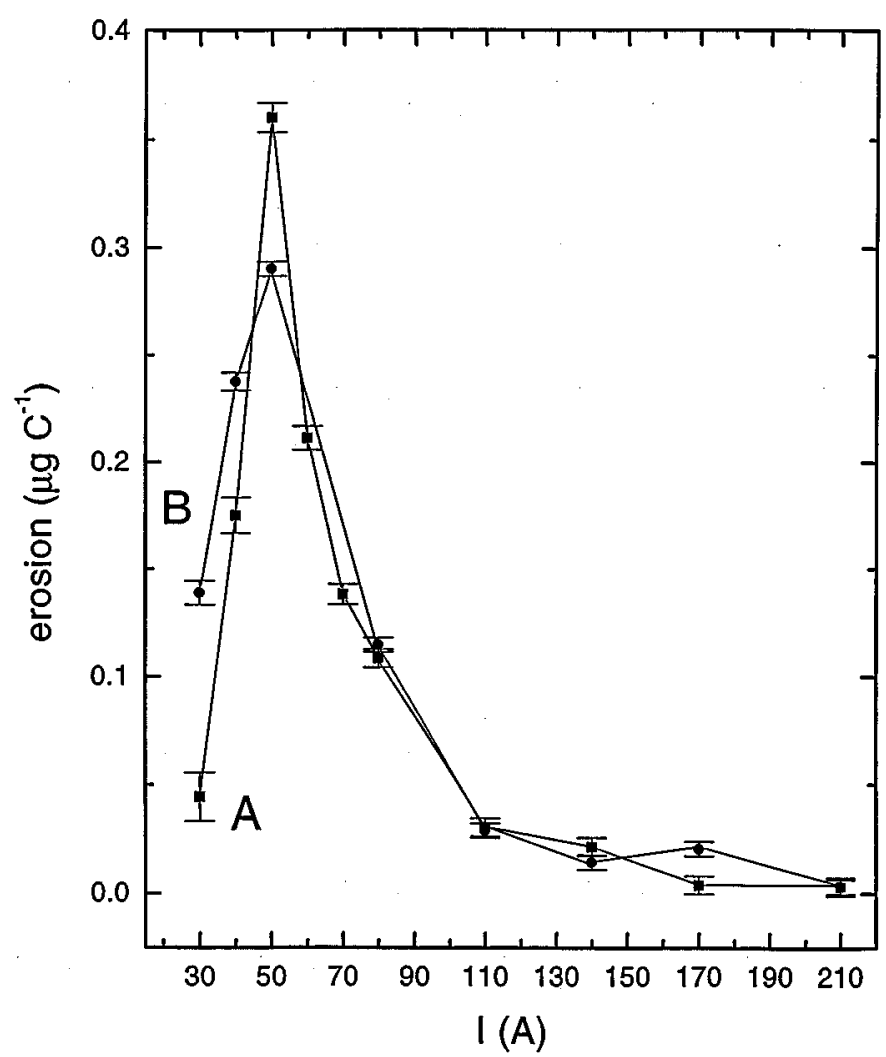

Fig. 3. Erosion dependence on arc current intensity for different arcing times. Curve A corresponds to $5 \mathrm{~min}$ arcing, while curve B corresponds to $10 \mathrm{~min}$ arcing.

\section{RESULTS}

The dependence of the erosion rate with the current intensity of the arc for different arcing times is shown in Fig. 3. To take the measurements, a separate cathode was used for each curve. Each one of these two cathodes was operated successively at the current intensities corresponding to the experimental points shown in the graph. The erosion rate for each current intensity was determined by the procedure indicated in the previous section. The uncertainty in the measurements is shown by the error bars on the experimental points in the figures. This uncertainty is determined by the precision of the instruments used. The values obtained on repeating the measurements for a given current did not show a significant dispersion of the measured erosion value.

It can be seen in Fig. 3 that at a critical current intensity $I_{C R}=50 \mathrm{~A}$ a maximum is reached in the erosion rate, which becomes about a factor of 10 greater than the erosion rate at high current intensities ( $I \geqq 140 \mathrm{~A}$ ). As shown in the figure, the value of $I_{C R}$ is the same for the two curves.

The results for the erosion rate shown in Fig. 4 were obtained using a new cathode for each current intensity. In this way, it was guaranteed that for each current intensity, the cathode surface was in similar conditions at the beginning of the cathode's work, since the cathode had not previously been used. Erosion was measured after $10 \mathrm{~min}$ of arcing at the corresponding current intensity.

In Fig. 4, it is seen again that a maximum of the erosion rate appears at the critical intensity of $50 \mathrm{~A}$, with a value of the erosion rate several times greater than that corresponding to high

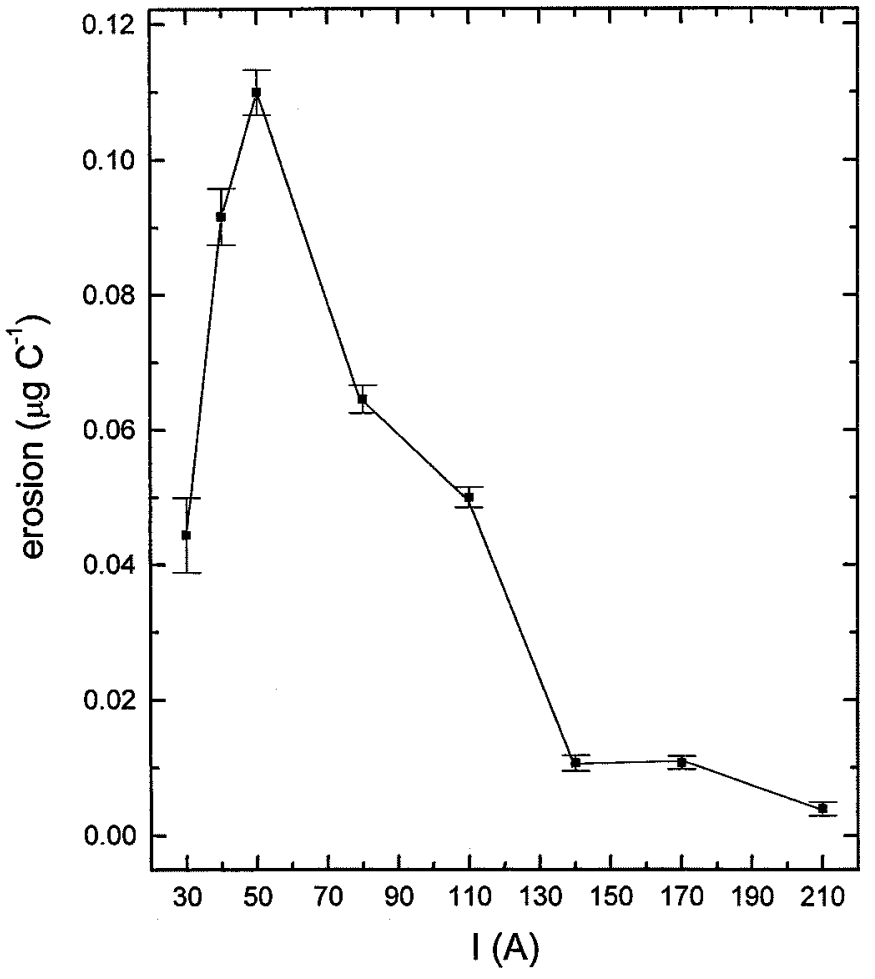

Fig. 4. Erosion dependence on current intensity obtained by using new cathodes for each current intensity. The arcing time was $10 \mathrm{~min}$.

current intensities. It is, therefore, assured that the maximum erosion found is related to the cathode functioning in the arc and not to the state of the cathode's surface before beginning the arc.

At low current intensities (up to $80 \mathrm{~A}$ ), the erosion values reported in Fig. 4 are less than those shown on the B curve in Fig. 3, which also correspond to an arcing time of $10 \mathrm{~min}$. This is due to the fact that in the measurements of this latter curve, a single cathode was used and thus, the cathode surface was already eroded by the previous use of the cathode. On these eroded surfaces, with craters and projections, intense fields occur that cause a greater erosion than on the smooth surfaces of the new cathodes. On the other hand, at high current intensities, the erosion rate values shown in Fig. 4 and on the B curve in Fig. 2 are similar, because high current intensities quickly provoke a great erosion on the cathode surface, and the difference between an initially new cathode surface and an already worn surface loses importance.

In Figs. 5-7, electron microscope images are shown of the tip cross-sections after arcing of three cathodes used to obtain the curve of Fig. 4. These cathodes operated at current intensities of 50, 80, and $170 \mathrm{~A}$, respectively, for $10 \mathrm{~min}$. On them can be seen the typical sponge-form structures caused by the processes of fusion, evaporation, and redeposition of cathode material that occur on the cathode surface during its working time. The EDX analysis of these cathodes revealed the total absence of thorium on the spot zones of the cathode, in agreement with the results reported in [14]. It should be pointed out that on cathodes operated at 80 and $170 \mathrm{~A}$, zones of the surface that remain unaltered, with the same morphological structure of a new cathode, appear 


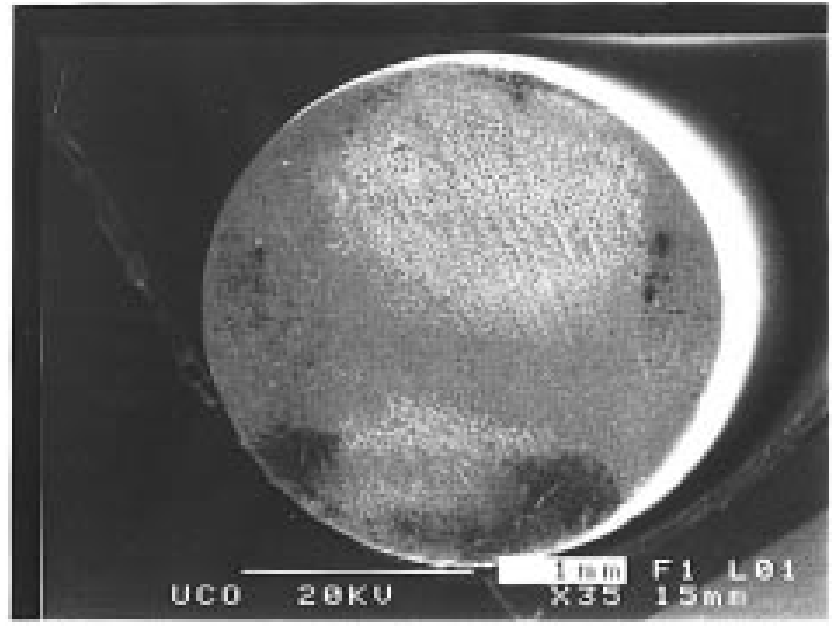

(a)

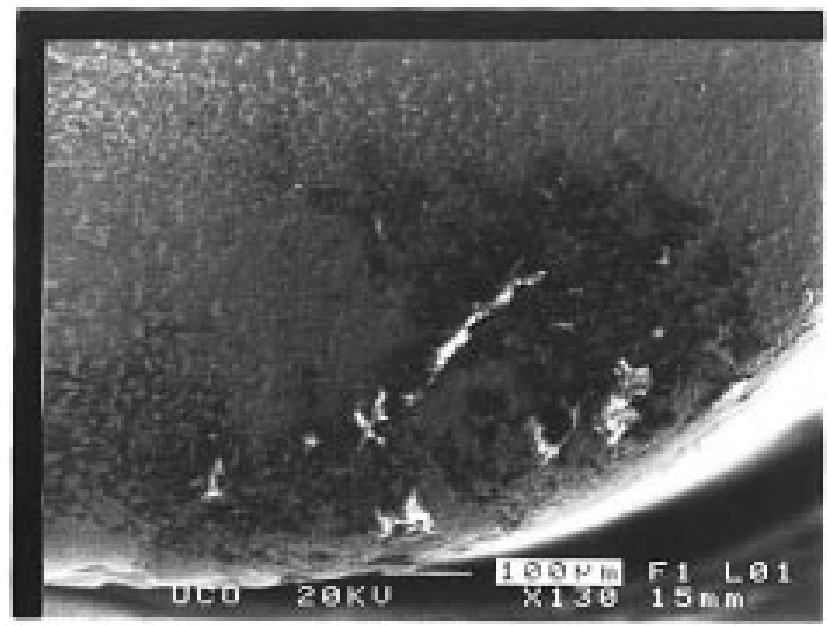

(b)

Fig. 5. SEM photos of cathode surface after arcing with an arc current of 50 A for $10 \mathrm{~min}$. (a) Surface of the cathode tip cross-section; (b) detail of bottom zone of the cathode tip surface.

[the lower left zone of the cathode cross-section in Fig. 6(A) and the left side of the cathode cross-section in Fig. 7(A)].

A total electric charge of $30 \mathrm{kC}$ passed through the cathode of Fig. 5 during arcing, since this cathode worked at the critical current intensity $I_{C R}=50$ for $10 \mathrm{~min}$. It is interesting to compare its surface with the surfaces of other cathodes through which the same amount of coulombs passed, but which were operated at current intensities different from $I_{C R}$. To make this comparison, two new cathodes were taken. One of them was operated for $375 \mathrm{~s}$ at an intensity of $80 \mathrm{~A}$ and the other for $1000 \mathrm{~s}$ at an intensity of $30 \mathrm{~A}$, so that a charge of $30 \mathrm{kC}$ passed through both cathodes. In Fig. 8, electron microscope images of the surfaces of these cathodes after arcing are shown. Once again, zones of the cathode surface that have not been altered by arcing appear. On the eroded regions of these cathodes, the morphological alterations of the surface are similar to the alterations of the cathode surface in Fig. 5. However, the worn area on the surface of the cathodes in Fig. 8 is less than the worn area of the cathode in Fig. 5, which operated at the critical current intensity. Although no quantitative conclusions can be drawn from this observation,

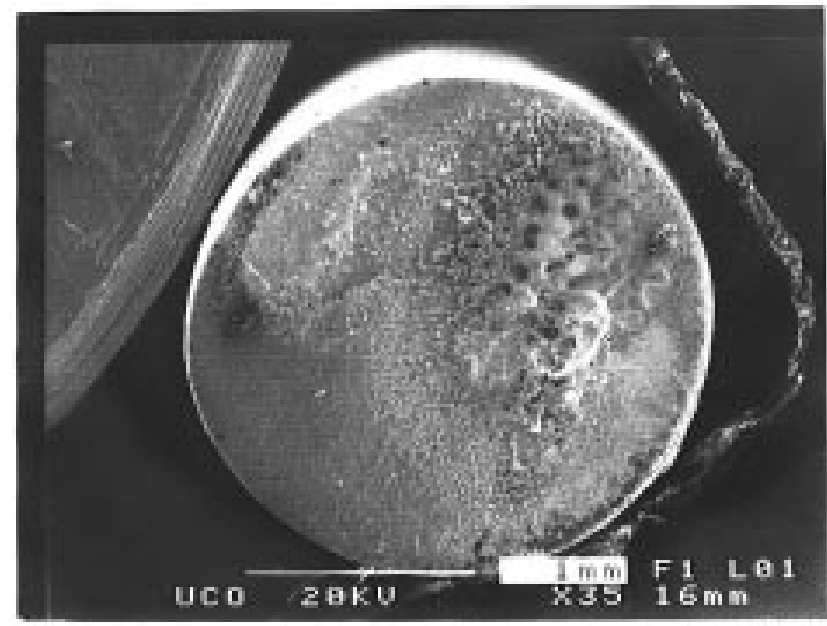

(a)

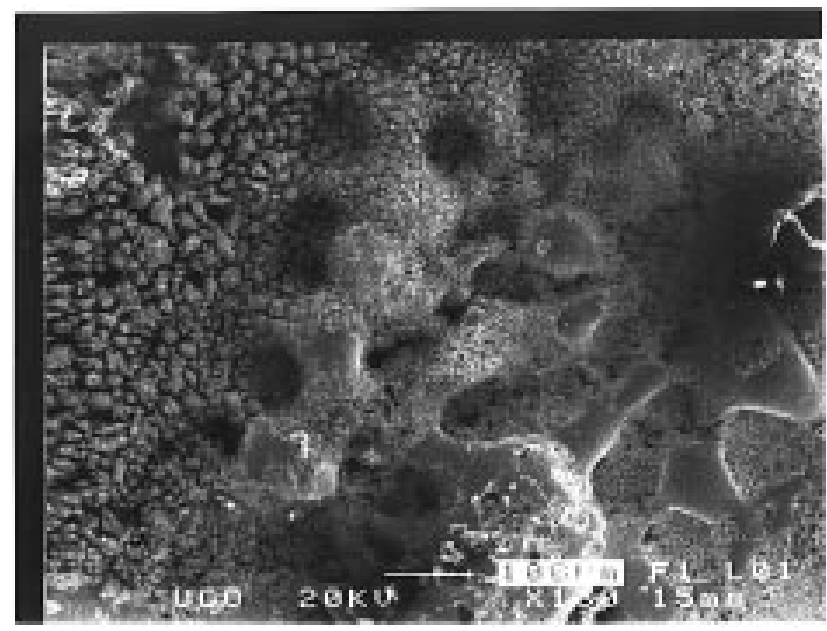

(b)

Fig. 6. SEM photos of cathode surface after arcing with an arc current of $80 \mathrm{~A}$ for $10 \mathrm{~min}$. (a) Surface of the cathode tip cross-section; (b) detail of upper right zone of the cathode tip surface.

it backs the measurements made of cathode mass loss that indicate a maximum erosion rate at $50 \mathrm{~A}$.

\section{DISCUSSION}

In thoriated tungsten cathodes, the value of the work function depends on the degree of spot coverage by thorium atoms. If the coverage is null, the value of the work function coincides with that of tungsten and very high temperatures are reached on the spot. If the coverage is maximum, the work function coincides with that of thorium and the cathode keeps working at relatively low temperatures, less than the tungsten melting temperature.

The degree of spot coverage by thorium atoms is the result of the balance between the arrival of thorium atoms to the spot by diffusion through the cathode bulk and the net evaporation of thorium atoms on the spot. This latter includes the processes of evaporation of thorium atoms and of the readsorption of a fraction of the evaporated atoms due to their ionization and return to the surface of the cathode under the action of the electric field on the cathode zone. The diffusion of thorium is strongly influenced by the temperature profile in the cathode, which depends on the arc current intensity [17]. 


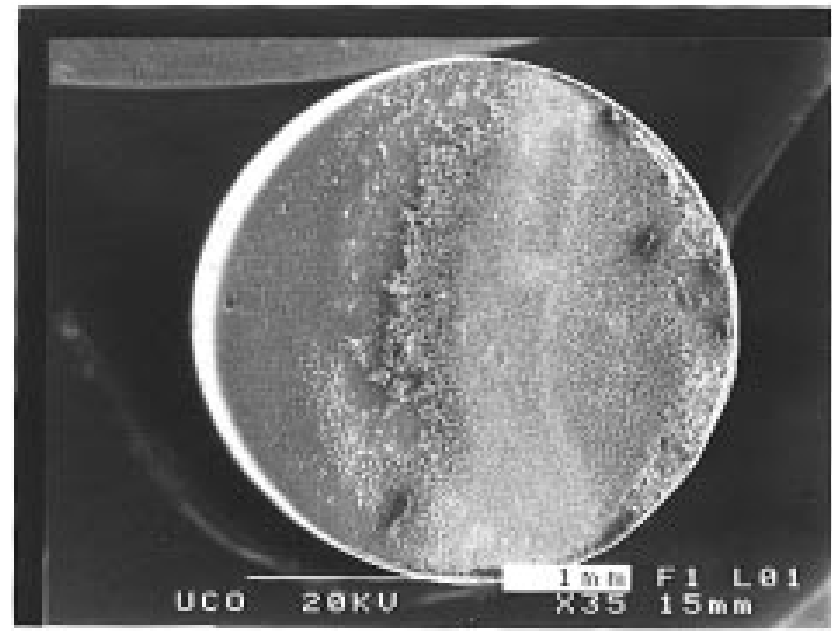

(a)

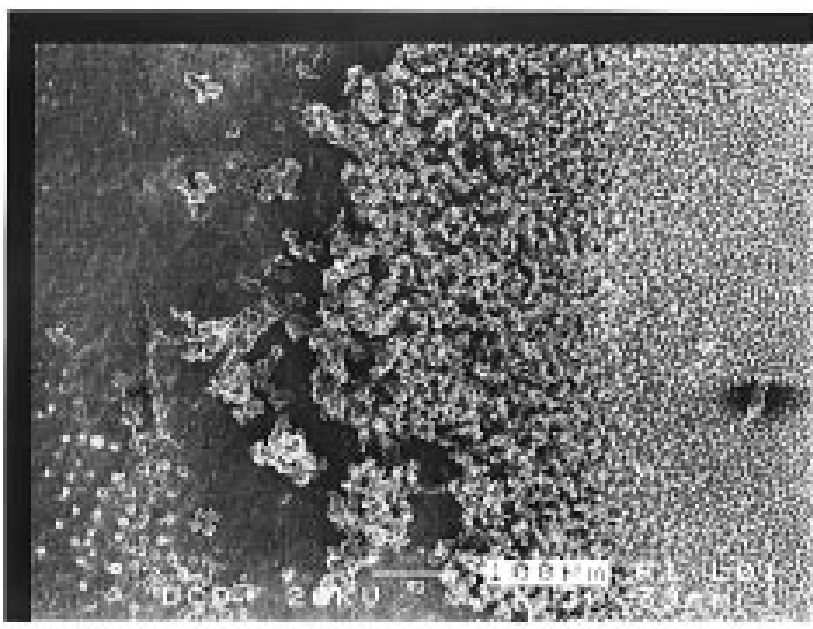

(b)

Fig. 7. SEM photos of cathode surface after arcing with an arc current of 170 A. (a) Surface of the cathode tip cross-section; (b) detail of central zone of the cathode tip surface.

At low current intensities, a low erosion in the cathode is expected. As the current increases, the spot temperature tends to increase for the thermionic emission must supply the required extra electrons. The raising of the spot temperature favors the desorption of the thorium atoms which increases the working function of the cathode. This process tends to augment erosion as the current intensity increases. However, the increase in current intensity also provokes a greater Joule heating in the cathode bulk. Therefore, the temperature distribution inside the cathode becomes more favorable for diffusion and the flux of thorium to the spot is increased. This process tends to diminish the working function. Therefore, both the spot temperature and the cathode erosion also tend to diminish. The overall effect of this two opposite processes might explain the existence of the critical current that we have experimentally found for which cathode erosion is maximum.

To determine the influence of the diffusion and desorption of thorium in erosion, it is interesting to compare the experimental results we obtained with similar erosion measurements made in pure tungsten cathodes. We attempted to measure the erosion rate in pure tungsten cathodes for various arc current intensi-

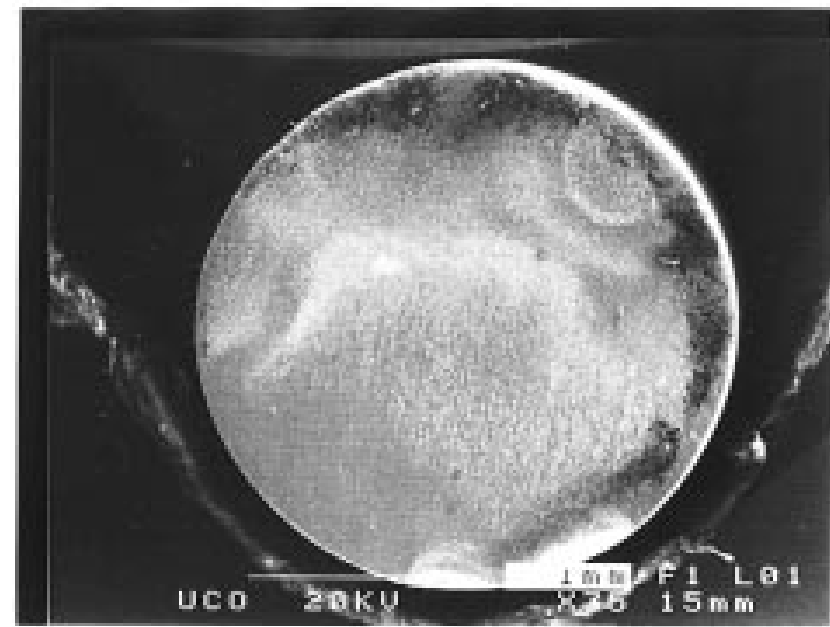

(a)

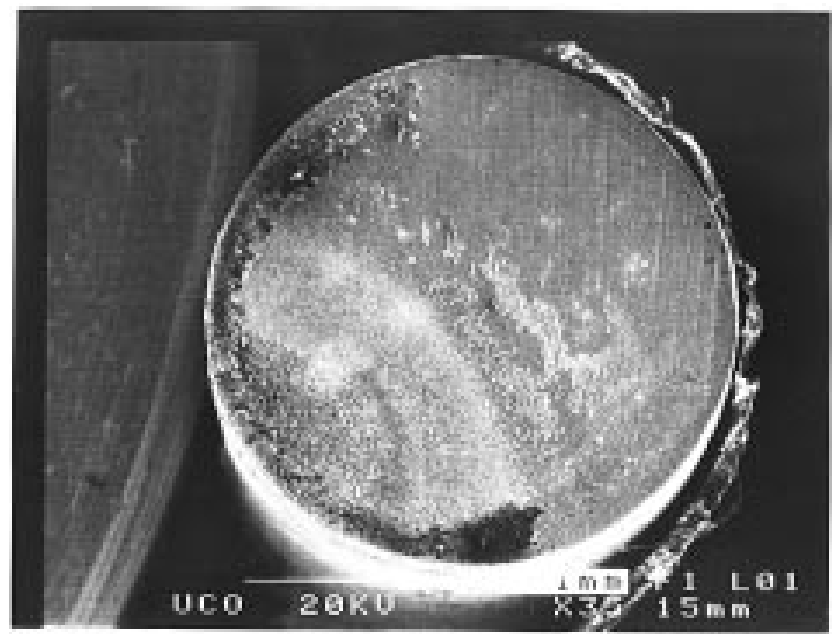

(b)

Fig. 8. SEM photos of the surfaces of two cathodes through each of which have passed $30 \mathrm{kC}$. (a) Cathode tip cross-section of cathode used for 1000 seconds with an arc current of $30 \mathrm{~A}$; (b) cathode tip cross-section of cathode used for 375 seconds with an arc current of $80 \mathrm{~A}$.

ties. Unfortunately, our measurement were unreliable, since a great dispersion of erosion rate values appeared for every current intensity. The same difficulty with pure tungsten cathodes was encountered in [15]. There, the problem was attributed to the influence of turbulent pressure fluctuations on the ejection of liquid metal droplets, which is the major erosion mechanism in pure tungsten cathodes.

One possibility that should be discussed is whether the results we show in Figs. 3 and 4 were due to the effect of erosion on the cathode edges. Since we used cylindrical cathodes with a flat tip, we had cathodes with sharp edges at the cathode end. If, at low current densities, the spot is located on the cathode's edges, abnormally high erosion could be obtained for these currents due to the sharp edge on the spot area. At high current densities, the spot could cover a greater area of the cathode's transversal section with which there would not be localized erosion at the sharp edges. The anomalous increase in erosion at low current intensities could provoke a peak in the erosion curve.

To investigate the influence of the sharp edge of the cathodes in our results, we have taken erosion measurements in a cathode 


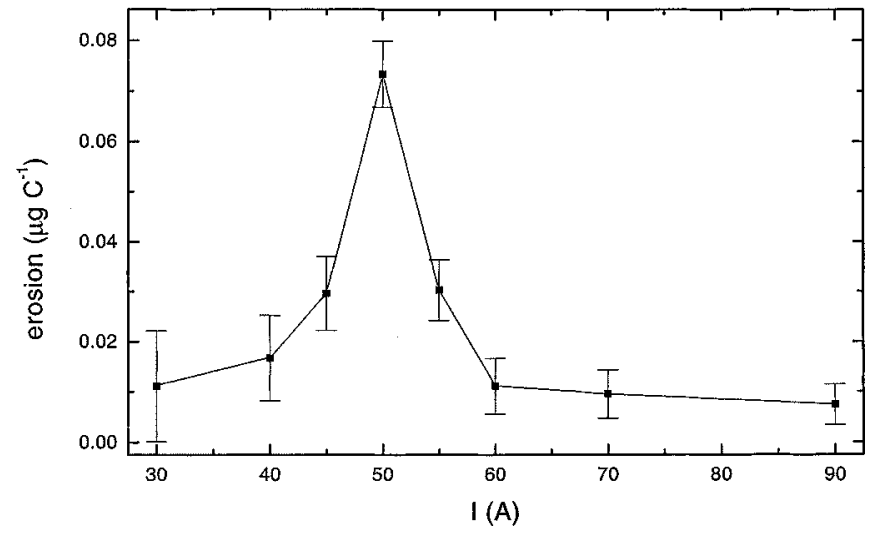

Fig. 9. Erosion dependence on arc current intensity in a cathode with rounded edges. The arcing time was $5 \mathrm{~min}$.

whose edges were rounded before arcing, thus eliminating its sharp edges. The results obtained are shown in Fig. 9. As can be seen, the curve of erosion as a function of the current intensity is similar to that obtained using flat tip cathodes. This indicates that the existence of a maximum in erosion for a critical current intensity is not originated by the presence of sharp edges on the cathode's tip.

Since we used graphite anodes, we should discuss the possibility of cathode contamination by carbon atoms and the influence that it may have on our results. The EDX analysis of the tip of all cathodes used shows the total absence of carbon on all points of the transversal section of the cathode tip. This occurs both in the cathodes used once to obtain the experimental points in Fig. 4, as well as in those used repeatedly to obtain the experimental points in Fig. 3. On the other hand, the EDX analysis of the lateral surface of a new cathode showed the following atomic composition: $3.22 \%$ oxygen, $1.87 \%$ thorium, $88.32 \%$ tungsten, and $6.58 \%$ carbon. The percentage of carbon on the lateral surface of another cathode after use in the arc with the mixture of argon and hydrogen as plasma-forming gas was $8.49 \%$ (see Fig. 2). This percentage of carbon is only slightly greater than that of a new cathode. Since the contamination of carbon atoms on the lateral surface of the cathodes that we used was little and since no contamination at all appeared on the active zones of the cathodes that we used, we conclude that the results obtained are related to the working of the thoriated tungsten cathodes and not to a possible contamination of them.

To verify that the carbon contamination was not responsible for the existence of the critical current, we repeated the erosion measurements with the previously explained experimental conditions, but using an iron anode instead of a graphite anode. The results obtained are shown in Fig. 10. This figure shows the presence of a critical current with the same value ( $I=50$ A) that was previously obtained. This proves that the results we obtained were not associated with cathode contamination.

\section{CONCLUSION}

The dependence of the erosion rate of a thoriated tungsten cathode on the arc current intensity has been experimentally investigated. It has been found that a critical value of current intensity, $I_{C R}$, exists for which the cathode erosion rate is max-

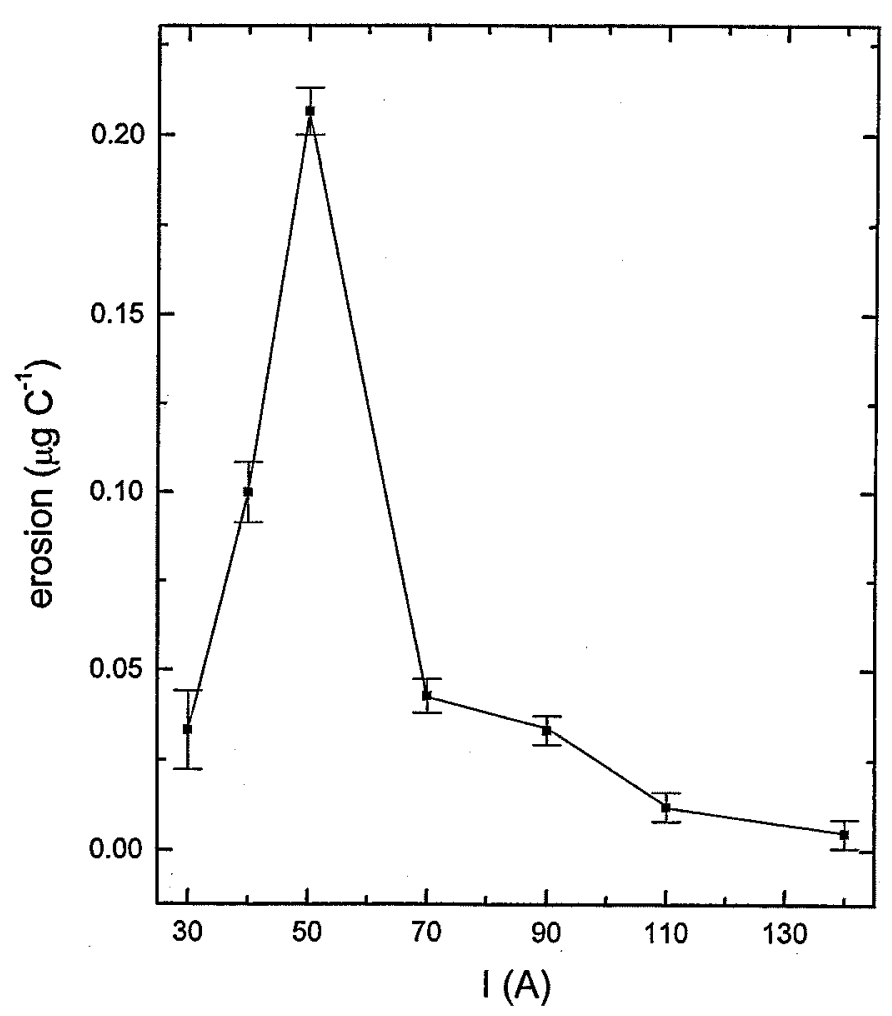

Fig. 10. Erosion dependence on arc current intensity in an arc with iron anode. The arcing time was 5 min.

imum. For greater values of current intensity, the erosion rate falls, tending to a constant value at high current intensities.

Although the concrete value of $I_{C R}$ depends on the arc characteristics (type of cathode, geometry of torch used, value of gas flux, etc.), it has been shown that the value of $I_{C R}$ under whatever concrete working conditions are used, can be experimentally determined in a simple way.

The determination of the $I_{C R}$ value is important from a practical point of view to avoid operation of the arc with current intensities that produce a maximum of cathode erosion and, consequently, shorten the useful lifetime of the cathode.

\section{ACKNOWLEDGMENT}

The authors wish to thank the services of the Electron Microscopy Unit of Cordoba University, Cordoba, Spain.

\section{REFERENCES}

[1] E. Pfender, "Thermal plasma technology: Where do we stand and where are we going?," Plasma Chem. Plasma Proc., vol. 19, pp. 1-31, 1999.

[2] J. Heberlein, "Electrode phenomena in plasma torches," Ann. NY Acad. Sci., vol. 891, pp. 14-27, 1999.

[3] P. Fauchais and A. Vardelle, "Thermal plasmas," IEEE Trans. Plasma Sci., vol. 25, pp. 1258-1280, Dec. 1997.

[4] S. Coulombe and J.-L. Meunier, "Theoretical prediction of nonthermionic arc cathode erosion rate including both vaporization and melting of the surface," Plasma Sources Sci. Technol., vol. 9, pp. 239-247, 2000.

[5] I. I. Beilis, "Application of vacuum arc cathode spot model to graphite cathode," IEEE Trans. Plasma Sci., vol. 27, pp. 821-826, 1999.

[6] P. Solana, P. Kapadia, and J. Dowden, "A mathematical analysis of heating effects and electrode erosion in conical electrical arc cathodes," J. Phys. D, Appl. Phys., vol. 31, pp. 3446-3456, 1998. 
[7] A. Marotta and L. I. Sharakhovsky, "Heat transfer and cold cathode erosion in electric arc heaters," IEEE Trans. Plasma Sci., vol. 25, pp. 905-912, Oct. 1997.

[8] L. I. Sharakhovsky, A. Marotta, and V. N. Borisyuk, "A theoretical and experimental investigation of copper electrode erosion in electric arc heaters-Part III: Experimental validation and prediction of erosion," J. Phys. D, Appl. Phys., vol. 30, pp. 2421-2430, 1997.

[9] P. J. Estrup, J. Anderson, and W. E. Danforth, "LEED studies of thorium adsorption on tungsten," Surf. Sci., vol. 4, pp. 286-298, 1966.

[10] B. Ya. Moizhes and V. A. Nemchinskii, "Vaporization rate of impurity and the operating conditions of an activated plasmotron cathode," Sov. Phys. Tech. Phys., vol. 29, pp. 612-616, 1984.

[11] V. A. Nemchinsky, "Life time of a refractory cathode doped with a work-function-lowering dopant," J. Phys. D, Appl. Phys., vol. 29, pp. 2417-2422, 1996.

[12] E. Casado and V. Colomer, "The numerical modelling of Joule heating effects in thoriated tungsten cathodes of high-current plasma arcs," $J$. Phys. D, Appl. Phys., vol. 33, pp. 1342-1347, 2000.

[13] P. Tsantrizos and W. H. Gauvin, "Cathode erosion phenomena in a transferred-arc plasma reactor," Plasma Chem. Plasma Process., vol. 12, pp. 17-33, 1992.

[14] X. Zhou, B. Ding, and J. V. R. Heberlein, "Temperature measurement and metallurgical study of cathodes in DC arcs," IEEE Trans. Comp., Packag., Manufact. Technol. A, vol. 19, pp. 320-328, Sept. 1996.

[15] X. Zhou and J. Heberlein, "An experimental investigation of factors affecting arc-cathode erosion," J. Phys. D, Appl. Phys., vol. 31, pp. 2577-2590, 1998

[16] M. Ushio, A. A. Sadek, and F. Matsuda, "Comparison of temperature and work function measurements obtained with different GTA electrodes," Plasma Chem. Plasma Process., vol. 11, pp. 81-101, 1991.

[17] J. Haidar and A. J. D. Farmer, "Surface temperature measurements for tungsten-based cathodes of high-current free-burning arcs," J. Phys. D, Appl. Phys., vol. 28, pp. 2089-2094, 1995.

[18] H. Zhang, Z. Yang, and B. Ding, "Characteristics of $\mathrm{W}-\mathrm{ThO}_{2}$ thermionic cathode activated with nanoscale oxide," IEEE Trans. Comp. Packag. Technol., vol. 22, pp. 455-459, Sept. 1999.

[19] S. W. H. Yih and C. T. Wang, Tungsten. Sources, Metallurgy, Properties, and Applications. New York: Plenum, 1979, p. 311.

[20] J. J. Lowke, R. Morrow, J. Haidar, and A. B. Murphy, "Prediction of gas tungsten arc welding properties in mixtures of argon and hydrogen," IEEE Trans. Plasma Sci., vol. 25, pp. 925-930, Oct. 1997.

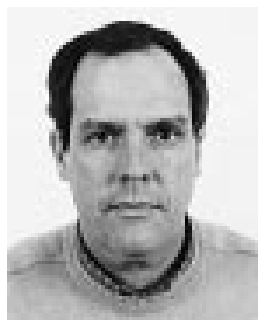

Eduardo Casado was born in Havana, Cuba in 1951. He received the M.S. and Ph.D. degrees in physics from the University of Havana, Havana, Cuba, in 1978 and 1991, respectively.

He has been a Staff Scientist at the Cuban Academy of Sciences and a Professor at the University of Havana. He is currently a Lecturer at the Cordoba University, Cordoba, Spain. His general research interests include plasma electrode interaction and thermal plasma applications.

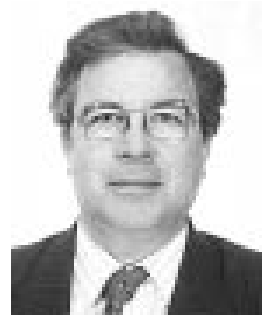

Vicente Colomer was born in Valencia, Spain in 1946. He received the M.S. and Ph.D. degrees from the Complutense University of Madrid, Spain, in 1969 and 1976, respectively.

He became an Assistant Professor at Complutense University in 1978 and a Full Professor at Cordoba University, Cordoba, Spain, in 1981. He was a visiting Scientist of the Plasma Fusion Center, Massachusetts Institute of Technology, Cambridge, MA, in 1981. From 1984 to 1990, he was Rector of the Cordoba University. He has been the Head of the Physics Department at Cordoba University since 1991. His research interests include electronegative plasmas, plasma sheath, and thermal plasmas applications.

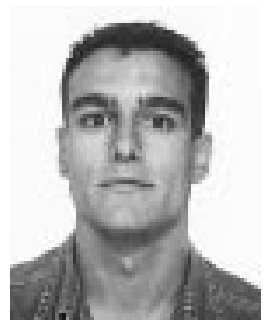

Rafael Sicilia was born in Cordoba, Spain, in 1972. $\mathrm{He}$ received the electrical engineering degree from the Cordoba University, Cordoba, Spain, in 1997.

Since 1998, he has been with the Plasma Technology Laboratory, Cordoba University. His research interests include the industrial applications of plasma torches.

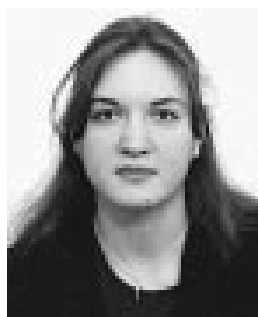

Encarnación Muñoz-Serrano was born in Cordoba, Spain, in 1977. She received the M.S. degree in physics from Cordoba University, Cordoba, Spain, in 2000. She is working toward the Ph.D. degree. 